\title{
Riesgos y salud ocupacional en el empleo público: Un análisis de perfiles psicosociales
}

\author{
Roxana Graciela Marsollier a y b ${ }^{1}\left(\mathbb{C}, \&\right.$ Cristián David Expósito a y b ${ }^{2}{ }^{2}$ \\ Consejo Nacional de Investigaciones Científicas y Técnicas de Argentina, \\ Mendoza, Argentina a ${ }^{\text {; }}$ Universidad Nacional de Cuyo, Mendoza, \\ Argentina ${ }^{\text {b3 }}$.
}

\section{RESUMEN}

La investigación que se reseña tuvo por finalidad identificar los perfiles psicosociales de trabajadores estatales a partir del modelo burnout-engagement y otros factores asociados. Participaron 147 empleados administrativos de un organismo público de Argentina. Se utilizó un diseño cuantitativo, de tipo descriptivo-explicativo. Se aplicaron distintas pruebas psicométricas para medir burnout, engagement, características de la tarea, clima psicosocial y percepción de acoso. Los resultados muestran la coexistencia de distintos perfiles psicosociales en el mismo ambiente laboral e informan la toma de decisiones de gestión, mediante acciones preventivas y/o correctivas de los riesgos en el trabajo, o bien, estrategias motivadoras que potencien la salud laboral. En el plano teórico, aporta a la perspectiva dialéctica de integrar experiencias positivas y negativas en una misma dinámica laboral.

Palabras Claves

empleo público; burnout; engagement; riesgos psicosociales; salud ocupacional.

\begin{abstract}
The objective of this study is to identify the psychosocial profiles of state employees based on the burnoutengagement model and other associated factors. 147 administrative staff of a Government Offices in Mendoza, Argentina participated. Different psychometric tests were applied to measure burnout, engagement, characteristics of the job, psychosocial climate and perception of mobbing. The methodological design is quantitative, descriptive-explanatory. The results obtained allow us to identify the coexistence of different psychosocial profiles in the same labor context, an important input for management decision making with a view to generating preventive and corrective strategies in relation to psychosocial risks at work or motivating occupational health. On the theoretical level, it contributes to the dialectical perspective of integrating positive and negative experiences in one same job dynamic.
\end{abstract}

Keywords

state employment; burnout; engagement; psychosocial risks; occupational health

\footnotetext{
1 Correspondence about this article should be addressed Roxana Graciela Marsollier: rgmarsollier@ conicet.gov.ar

2 Conflicts of Interest: The authors declare that the research was conducted in the absence of any commercial or financial relationships that could be construed as a potential conflict of interest.

${ }^{3}$ Centro de Investigaciones Cuyo "Dr. Abelardo Pithod"
} 
Risks and occupational health in state employment: analysis of coexisting psychosocial profiles

Las investigaciones en psicología laboral centraron sus primeros estudios en la identificación de aquellos factores que originan los procesos de estrés o malestar en las organizaciones. El estrés en general, y particularmente, el desgaste profesional, han sido abordados científicamente en casi todos los contextos laborales, considerándose problemáticas transculturales, por haber sido identificadas en distintas culturas y tipos de trabajo (Gil Monte, 2007; Salanova \& Llorens, 2008). Sin embargo, con el surgimiento de la psicología positiva, comenzó a ponerse el acento en otros factores tales como valores, recursos personales, motivación, compromiso laboral, etc., en tanto actúan como protectores frente a los riesgos laborales (Bakker \& Rodríguez-Muñoz, 2012). Sin pretender dejar de lado el enfoque tradicional, esta nueva mirada aportó conocimientos complementarios, que muestran otras formas en las que puede comprenderse la relación de las personas con su trabajo. De hecho, la psicología de la salud ocupacional, surgida en el marco de la psicología positiva, contempla la necesidad de incluir tanto los aspectos negativos como los positivos en el análisis de las organizaciones, en tanto afectan el comportamiento de las personas en su trabajo e incluso, fuera de él (Salanova et al., 2014).

En otros términos, las experiencias laborales positivas y negativas, no pueden ser abordadas de manera aislada y descontextualizada, ya que forman parte de una misma realidad integral, cambiante e incierta. En este sentido, los ambientes laborales pueden ser caracterizados como contextos complejos donde confluyen aspectos estructurales, relaciones sociales y procesos personales, en una dinámica de particular interacción. En este marco, el empleo público reviste especial interés, por las peculiares características que reúnen los organismos estatales en cuanto a su estructura fuertemente burocratizada y verticalista que traen aparejadas monotonía y falta de autonomía en la tarea, con su correspondiente impacto en el comportamiento y rendimiento de sus trabajadores (Marsollier, 2018).

En el presente estudio buscamos comprender, por qué frente a un mismo ambiente de trabajo pueden identificarse diversas percepciones, actitudes y comportamientos de los empleados, que incluso, pueden llegar a ser opuestos. En otros términos, analizar por qué en un mismo contexto de empleo público, algunos trabajadores se sienten satisfechos y motivados, mientras otros, se perciben molestos y desgastados por su tarea.

Tomamos como eje de nuestra investigación el modelo burnout/engagement (Salanova et al., 2000) e incorporamos otras variables que resultaron ser mediadoras de 
estos factores en análisis previos, tales como a) características de la tarea, b) clima psicosocial de trabajo, y c) percepción de acoso laboral. Veamos algunas nociones teóricas que nos permiten fundar nuestra propuesta.

\section{Modelo burnout-engagement}

Si bien surgieron en épocas y contextos diferentes, desde el punto de vista teórico, burnout y engagement son conceptos opuestos y representan realidades laborales antagónicas. El burnout, ha sido estudiado por más de cuatro décadas ya, en tanto constituye una problemática claramente presente en todos los contextos laborales y en diferentes culturas, por lo que su estudio se extendió rápidamente a nivel mundial (Schaufeli et al., 2009). Pasaron muchos años hasta el surgimiento del engagement, noción que alude al compromiso laboral o vinculación psicológica con el trabajo. Este constructo, surge en el marco de la Psicología Positiva, movimiento que cobra fuerza en los '90 y centra su atención en las fortalezas y potencialidades de las personas en el trabajo (Seligman \& Csikszentmihalyi, 2000). El engagement es definido como un estado mental positivo en relación con el trabajo que se caracteriza por el vigor, la dedicación y la absorción puesta en la tarea y constituye un concepto específico e independiente del burnout (Schaufeli et al., 2002).

Así, el binomio burnout-engagement, se presenta como un modelo de análisis teórico de distintas experiencias psicosociales que pueden desarrollarse en un mismo ambiente de trabajo. A nivel teórico, existen dos tendencias que intentan explicar la vinculación entre ambos constructos. La primera de ellas, sugiere que esta relación puede ser entendida como un continuum entre dos extremos opuestos, donde el engagement simboliza un objetivo a conseguir cuando tiene lugar la intervención en situaciones de desgaste (Maslach \& Leiter, 2008). De hecho, desde sus mismos orígenes, la medición del burnout, -mediante el Maslach Burnout Inventory en sus distintas versiones-, contempla la existencia contrapuesta de las experiencias negativas de cansancio emocional y cinismo -conocidas como el corazón del burnout-, con la eficacia profesional, como dimensión positiva vinculada al trabajo (Maslach et al., 1996). Es decir, en un cuadro típico de desgaste, es esperable encontrar altos niveles de las dos primeras, lo que implicaría bajas puntuaciones en eficacia profesional. El modelo burnout-engagement advierte esta idea de un polo positivo contrapuesto a las dimensiones constitutivas del desgaste, entendiendo que, para los casos de burnout, la energía se convertía en agotamiento, la participación en cinismo y la eficacia en ineficacia (Maslach 
\& Leiter, 1997). Posteriormente, los estudios de Leiter y Maslach (2016) proponen la existencia de un abanico más amplio de experiencias entre ambos extremos, los que configuran distintos perfiles psicosociales latentes: desgastado (cuadro típico de burnout), desinteresado (cuando predomina el cinismo), sobreexigido (caracterizado por el agotamiento psicofísico excesivo), ineficiente (ante la baja eficacia profesional) o comprometido (al presentar altos niveles de engagement).

Asimismo, Leiter y Maslach (2017) sostienen que estar en extremos diferentes no significa que sean constructos excluyentes y que una posible diferencia entre ambos, simplemente, radica en la variable tiempo. Al respecto, cabe destacar que, desde sus primeros estudios, ambas experiencias fueron consideradas como constantes en el tiempo. Así, por ejemplo, el engagement fue identificado como un estado cognitivo y emocional de vinculación positiva con el trabajo, persistente en el tiempo y de carácter estable (Salanova et al., 2000). Sin embargo, los estudios posteriores de Sonnetang y Kühnel (2016) encontraron evidencia empírica que demuestra que la experiencia positiva de compromiso puede diferir día a día, incluso puede variar en distintos momentos de una misma jornada de trabajo. No obstante, las investigaciones sobre burnout, continúan considerando su persistencia en el tiempo, entendiendo al síndrome como una respuesta prolongada a estresores interpersonales crónicos presentes en el contexto de trabajo (Maslach, 2009).

La segunda tendencia en cuanto a la compresión de la vinculación burnoutengagement, concibe a ambos procesos como totalmente independientes entre sí, entendiendo que la ausencia de una experiencia de desgaste, por ejemplo, no implica necesariamente la existencia de una vivencia engaget, y viceversa (Salanova \& Llorens, 2008). En este sentido, se plantea que entre ambos constructos se da un movimiento espiralado, que puede ser descendente, si está teñido de experiencias negativas de desgaste; o bien ascendente, si predominan las vivencias positivas de compromiso laboral (Salanova et al., 2005).

Algunos estudios más recientes sostienen que, entre burnout y engagement, existiría una relación dialéctica que permite integrar la particular dinámica entre ambas experiencias, más allá de sus posibles diferencias y contradicciones (León et al., 2015). En este sentido de integración Schaufeli y De Witte (2017) postulan la existencia de una realidad dual entre ambas vivencias, entendiendo que no es posible afirmar que sean experiencias totalmente opuestas ni totalmente independientes. 


\section{Factores asociados}

A continuación, comentaremos algunas nociones generales sobre nuestras variables moduladoras: características de la tarea, clima psicosocial en el trabajo y acoso laboral.

En cuanto al análisis de las características que tiene la propia tarea, cabe destacar que distintos autores han analizado la influencia de experiencias negativas o positivas que se asocian a la labor cotidiana. Gil Monte (2012), analiza las disfuncionalidades de la tarea en términos de potenciales riesgos psicosociales que pueden derivar en ulteriores cuadros de desgaste. Entre los distintos aspectos señalados por el autor se encuentran la sobrecarga de trabajo - cuantitativa y/o cualitativa-, la monotonía y la escasa complejidad de la tarea o la falta de autonomía, que puntualmente son objeto de nuestro análisis.

En relación con la sobrecarga de trabajo, podemos afirmar que tradicionalmente ha sido un factor vinculado con el agotamiento emocional (Gil Monte et al., 2008; Pérez, 2013). Sin embargo, estudios efectuados en una población similar a la nuestra, sostienen que en muchos casos los empleados públicos más bien desearían una mayor carga laboral, ya que se sienten subvalorados en sus capacidades (Pérez Rubio \& Robina Ramírez, 2002). En relación con ello, podemos agregar que algunas características particulares que se atribuyen al empleo público, tales como la monotonía y escasa complejidad de la tarea, han sido asociadas con el aburrimiento o boredom, problemática causada cuando la tarea asignada se encuentra por debajo de las capacidades del trabajador, lo que afecta la motivación del empleado debido a una subestimulación (Schaufeli \& Salanova, 2014). Estas condiciones podrían favorecer el desarrollo del síndrome de boreout, que implica sentirse aburrido, infraexigido y desinteresado por el trabajo (Rothlin \& Werder, 2011). Otra de las características de la tarea es la autonomía, factor asociado en diversos estudios de manera directa con las experiencias positivas de engagement y autoeficacia laboral (Bakker \& Demerouti, 2013). Sin embargo, puntualmente, en el caso del empleo público, la falta de autonomía es considerada como uno de los principales riesgos psicosociales (Gil Monte et al., 2016). Por otra parte, estudios recientes indican que los niveles de engagement pueden modificarse según las características específicas de la tarea puntual que se esté desarrollando, mientras que los niveles de burnout tienden a mantenerse más allá de las variaciones en las tareas cotidianas (Sonnetang, 2017).

En cuanto al clima organizacional, existen abundantes investigaciones que analizan los conflictos interpersonales, la falta de apoyo social, de reconocimiento y de 
justicia organizacional como factores asociados al surgimiento del burnout (Golembiewski et al., 1983; Leiter \& Maslach, 1999; Winnubst, 1993). Pero además, el clima humano ha sido estudiado desde una perspectiva positiva de asociación con la motivación y el compromiso en el trabajo (Boada i Grau et al., 2004; Pecino et al., 2019). En este sentido, el engagement puede ser entendido como un fenómeno psicosocial colectivo que, mediante la interacción laboral cotidiana, influye positivamente en el desempeño de los trabajadores y contribuye a incrementar prácticas organizacionales saludables (Torrente et al., 2017).

Tanto los factores negativos como los positivos se encuentran presentes en todos los contextos laborales e influyen en el bienestar y salud de los empleados y en el funcionamiento organizacional. Los factores negativos son identificados como riesgos psicosociales y representan demandas del contexto, frente a las cuáles, los trabajadores buscarán el equilibrio o regulación haciendo uso de sus recursos personales, sociales y contextuales (Cifre et al., 2009). En este sentido, recursos tales como la autonomía, la autoeficacia, así como los aspectos colectivos de apoyo social y buenas relaciones en el trabajo, tendrían un efecto amortiguador de las demandas laborales negativas que llevan al desgaste profesional (Orgambídez-Ramos et al., 2015).

Finalmente, y en lo concerniente a la posible asociación con el acoso laboral, cabe señalar que el mobbing constituye una experiencia negativa en el trabajo, diferente al síndrome de burnout; ambos peligrosos riesgos de los contextos laborales actuales. El mobbing ha sido concebido como un proceso de maltrato verbal o modal, de carácter continuo por parte de una persona o grupo hacia un compañero, con la finalidad de destruirlo psicológicamente y obtener su salida de la organización (Fidalgo \& Piñuel, 2004). Diversos investigadores han profundizado las posibles causas del acoso, identificando en sus estudios factores personales, interpersonales y/o contextuales que mostraron estar asociados (Moreno Jiménez et al., 2005; Meseguer et al., 2007; Rodríguez Muñoz et al., 2009). Por otra parte, Olmedo y González (2006) sostienen que las organizaciones públicas generan mayores probabilidades de mobbing, debido a las escasas evaluaciones objetivas y a la influencia de las relaciones interpersonales con compañeros y jefes, en la promoción de la carrera laboral. Algunos estudios muestran interesantes vinculaciones entre burnout y mobbing, señalando mayores niveles de desgaste en quienes se consideran víctimas de acoso laboral (González Trijueque \& Delgado Marina, 2008). Específicamente, el mobbing se encontraría asociado al desgaste en sus dimensiones centrales: el agotamiento emocional y el cinismo (García Izquierdo 
et al., 2006). No obstante, los estudios no son concluyentes, ya que no siempre se pone en evidencia una relación directa entre ambas experiencias negativas, lo que pondría el acento en otras variables contingentes (Ayuso Marente \& Guillén Gestoso, 2008).

\section{Método}

\section{Diseño}

Utilizamos un diseño metodológico cuantitativo, de tipo descriptivo/explicativo, de corte transversal.

\section{Participantes}

Trabajamos con un muestreo intencional de empleados administrativos $(n=147)$ insertos en un organismo público de la Provincia de Mendoza, Argentina $151.7 \%$ hombres). Los participantes debían cumplir con el criterio de formar parte de la planta permanente o contratada con prestación efectiva de servicio en el sector administrativo. Este grupo se caracteriza por ser el staff que permanece estable, más allá de los cambios de la gestión política de turno. El promedio de edad al momento del relevamiento fue de 43.88 años ( $\mathrm{DE}=11.95)$, con una antigüedad de 16.07 años como empleado del estado $(\mathrm{DE}=10.95)$ y una media de 12.80 años ocupando el mismo puesto laboral $(\mathrm{DE}=10.90)$.

\section{Instrumentos}

Aplicamos un cuestionario semiestructurado destinado a recolectar datos cuantitativos y cualitativos. En la instancia cuantitativa, se recogieron variables sociodemográficas (edad, sexo, antigüedad, nivel de instrucción, profesión, situación laboral, entre otras) y se aplicaron distintas pruebas psicométricas. A continuación, presentamos una breve descripción de cada test y los índices de confiabilidad para esta muestra:

a) El Maslach Burnout Inventory - General Survey (MBI-GS) (Schaufeli et al., 1996).

Esta versión del MBI corresponde a la medición del desgaste profesional en personal de servicios generales, en este caso, empleados administrativos. La prueba consiste en una escala Likert de 16 ítems con siete grados de respuesta-de 0 (Nunca) a 6 (Todos los días) - mediante la cual, los participantes evalúan con qué frecuencia han experimentado distintas experiencias laborales. El instrumento, permite obtener puntuaciones independientes para sus tres factores: Cansancio emocional (5 ítems, $\alpha$ $=.826$, e. g., Me siento emocionalmente agotado por mi trabajo); el Cinismo (5 ítems, 
$\alpha=.790$, e. g., Sólo quiero hacer mi trabajo y que no me molesten) y por último, la Eficacia profesional (6 ítems, $\alpha=.743$, e. g., He realizado muchas cosas valiosas en mi trabajo).

b) Utrech Work Engagement Scale - UWES (Schaufeli et al, 2002). Mediante esta escala de 17 ítems, los participantes indican la frecuencia con que experimentan distintos sentimientos respecto de su trabajo, evaluando mediante una escala Likert que va de 0 (nunca) a 6 (todos los días). Nuestro análisis indica elevados niveles de consistencia interna del instrumento, tanto para sus tres subescalas: Vigor (6 ítems; $\alpha=.775$; e.g., En mi trabajo me siento lleno de energía); Dedicación, (5 ítems; $\alpha=.787$; e.g., Estoy orgulloso del trabajo que hago) y Absorción (6 ítems; $\alpha=.716$; e.g., Me es difícil "desconectarme" del trabajo), como en su consideración de medida global UWES $(\alpha=.898)$.

c) Cuestionario de Clima Psicosocial (basado en Martínez Gamarra \& Ros Mar, 2010; Moreno Jiménez, 1994). Se trabajó con una selección de seis dimensiones compuesta por 4 ítems cada una, que influyen en el clima humano dentro de las organizaciones, obteniendo resultados altamente confiables para cada uno de los factores analizados: a) Apoyo ( $\alpha=.826$; e.g., La colaboración entre compañeros suele ser habitual); b) Cohesión ( $\alpha=.749$; e.g., Las relaciones laborales suelen ir más allá del trabajo); c) Supervisión ( $\alpha=.888$; e.g., La supervisión del trabajo del grupo es eficaz); d) Equidad ( $\alpha=.788$; e.g., El reconocimiento del aporte que cada uno hace en el trabajo suele ser justo); e) Comunicación ( $\alpha=.771$; e.g., Normalmente me siento informado de lo que ocurre en la empresa) y e) Tolerancia ( $\alpha=.715$; e.g., Se aceptan las diferencias en la forma de trabajar).

d) Cuestionario sobre características de la tarea, es un instrumento elaborado ad hoc cuya finalidad es identificar las particularidades del quehacer administrativo en los organismos públicos. Posee 16 ítems, con una escala Likert de 5 grados -de 1: Totalmente en desacuerdo a 5: Totalmente de acuerdo-, mediante la cual el participante valora distintas situaciones laborales cotidianas. Para medir sus cualidades psicométricas, se efectuó un análisis factorial exploratorio con rotación Varimax. El índice de adecuación de la muestra $(\mathrm{KMO}=.708)$ y la prueba de esfericidad de Bartlett $\left(\chi^{2}\right.$ Bartlett $\left.=628,207, \mathrm{p}=.00\right)$ indican adecuados criterios empíricos para realizar el estudio. Este análisis permitió identificar tres factores, que explican el 47,74\% de la varianza total: a) Carga de trabajo ( 5 ítems, $\alpha=.746$; e. g., Realizo demasiadas tareas en función del horario laboral); b) Aspectos positivos de 
la tarea: tales como autonomía o sentirse motivado (4 ítems, $\alpha=.634$; e. g., Puedo decidir cómo hacer mi trabajo); y c) Aspectos negativos de la tarea; alude al aburrimiento o ciertas desventajas laborales (7 ítems, $\alpha=.606$; e. g., Mi trabajo me produce aburrimiento o El circuito burocrático entorpece el desarrollo de mi trabajo). Para el factor b) Aspectos positivos de la tarea y para el factor c) Aspectos negativos de la tarea se realizó un análisis de homogeneidad de los ítems mediante el coeficiente de correlación de Pearson para ambos factores encontrando que todos los ítems son estadísticamente significativos a nivel .01.

e) Negative Acts Questionnaire - NAQ-RE (Saez et al., 2003). Dicho instrumento consta de 24 reactivos. A fin de probar la validez del constructo de la escala, se llevó a cabo un análisis factorial exploratorio con rotación Varimax. El índice de adecuación de la muestra $(\mathrm{KMO}=.850)$ y la prueba de esfericidad de Bartlett $\left(\chi^{2}\right.$ Bartlett $=1724,261$; $\mathrm{p}=.00$ ) indican adecuados criterios empíricos. En el análisis factorial, se identifican dos factores relacionados con el mobbing a saber: a) Acoso laboral: incluye todos los modos de acoso relacionados con la propia tarea cotidiana, tanto en cuanto a la asignación de tareas, el resultado de los mismos y la relación profesional con colegas y compañeros (15 ítems; $\alpha=.898$; e.g., Se le ha privado de responsabilidad, asignándole tareas absurdas o inútiles); y b) Acoso personal: implica agresiones directas a la vida personal, tanto a nivel psicológico como social, pasando por críticas, amenazas, burlas, represalias, etc. ( 9 ítems; $\alpha=.810$; e.g., Se siente objeto de burlas o bromas de mal gusto).

\section{Procedimiento y análisis de datos}

Luego de obtener las autorizaciones correspondientes para ingresar al organismo público, realizamos el trabajo de campo oficina por oficina, previo consentimiento del jefe intermedio. El relevamiento fue efectuado en horario laboral a los empleados que luego de conocer el propósito del estudio, decidieron voluntariamente participar del mismo. Cabe señalar que en todas las etapas de este estudio se han resguardado los aspectos éticos, respetando el anonimato de los participantes y su participación voluntaria, bajo expreso consentimiento informado. Los datos fueron trabajados en distintos niveles de complejidad estadística mediante el software IBM $^{\circledR}$ SPSS $^{\circledR} 19.0$, con análisis descriptivos, correlacionales y por conglomerados de k medias. En relación con el análisis por conglomerados, se tuvo en cuenta que la solución convergiera en no más 
de 10 iteraciones y que los conglomerados propuestos presentaran diferencias significativas.

\section{Resultados}

\section{Indicadores descriptivos}

En la Tabla 1 se presenta una síntesis de los resultados descriptivos de las variables abordadas. Cabe señalar que, al tratarse de instrumentos con diferentes medidas, se optó por llevar las mediciones a escala 10 a fin de facilitar su tratamiento conjunto.

Tabla 1

Descriptivo de los factores analizados según sus variables constitutivas

\begin{tabular}{lllll}
\hline & M & DE & Mín. & Máx. \\
\hline 1. Cansancio emocional & 2.92 & 1.94 & .00 & 9.00 \\
2. Cinismo & 2.22 & 1.83 & .00 & 9.00 \\
3. Eficacia profesional & 7.52 & 1.39 & 2.00 & 9.00 \\
\hline 4. Vigor & 6.87 & 1.50 & 1.43 & 8.57 \\
5. Dedicación & 6.69 & 1.68 & 1.14 & 8.57 \\
6. Absorción & 5.83 & 1.64 & .71 & 8.57 \\
\hline 7. Carga de trabajo & 5.23 & 1.61 & 2.00 & 10.00 \\
8. Aspectos positivos de la tarea & 6.86 & 1.74 & 2.00 & 10.00 \\
9. Aspectos negativos de la tarea & 4.49 & 1.27 & 2.00 & 8.00 \\
\hline 10. Apoyo & 7.86 & 1.71 & 2.00 & 10.00 \\
11. Equidad & 6.75 & 1.95 & 2.00 & 10.00 \\
12. Comunicación & 7.05 & 1.74 & 2.00 & 10.00 \\
13. Tolerancia & 7.16 & 1.51 & 2.00 & 10.00 \\
14. Cohesión & 7.87 & 1.55 & 2.00 & 10.00 \\
15. Supervisión & 7.16 & 2.07 & 2.00 & 10.00 \\
\hline 16. Acoso laboral & 3.45 & 1.32 & 2.00 & 8.00 \\
17. Acoso personal & 2.42 & .74 & 2.00 & 6.22 \\
\hline No Mara
\end{tabular}

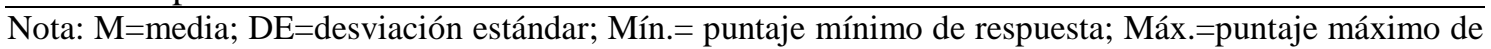
respuesta.

En relación con el resto de las variables, las subescalas de burnout, presentan un promedio relativamente bajo para sus dimensiones claves: Cansancio emocional $(\mathrm{M}=2.92)$ y Cinismo $(\mathrm{M}=2.22)$; mientras que la Eficacia profesional adquiere un valor promedio elevado $(\mathrm{M}=7.52)$.

Por su parte, el Engagement muestra puntuaciones media-altas en sus tres dimensiones, siendo un poco inferiores para la Absorción.

En relación con el resto de las variables observamos que la distribución de los valores medios de las características de la tarea, indican valores cercanos al percentil 50 para sus tres factores. 
En cuanto al clima psicosocial de trabajo, se observan valores promedios superiores a 7 puntos en todas sus escalas, siendo levemente inferior para la dimensión Equidad $(M=6.75)$.

Finalmente, en cuanto al acoso percibido, si bien los valores promedios son relativamente bajos, es igualmente preocupante, ya que atañe directamente a la integridad de algunos empleados que poseen una sensación de acoso laboral $(\mathrm{M}=3.45)$ y/o personal $(\mathrm{M}=2.42)$.

\section{Análisis de correlaciones}

En un nivel de análisis bivariado, las variables en análisis mostraron estar relacionadas entre sí. Consideraremos estas correlaciones como aceptables cuando la correlación de Pearson supere el valor de .30 (Aron \& Aron, 2002).

Tabla 2

Correlaciones entre MBI-G,. UWES y otros factores de estudio

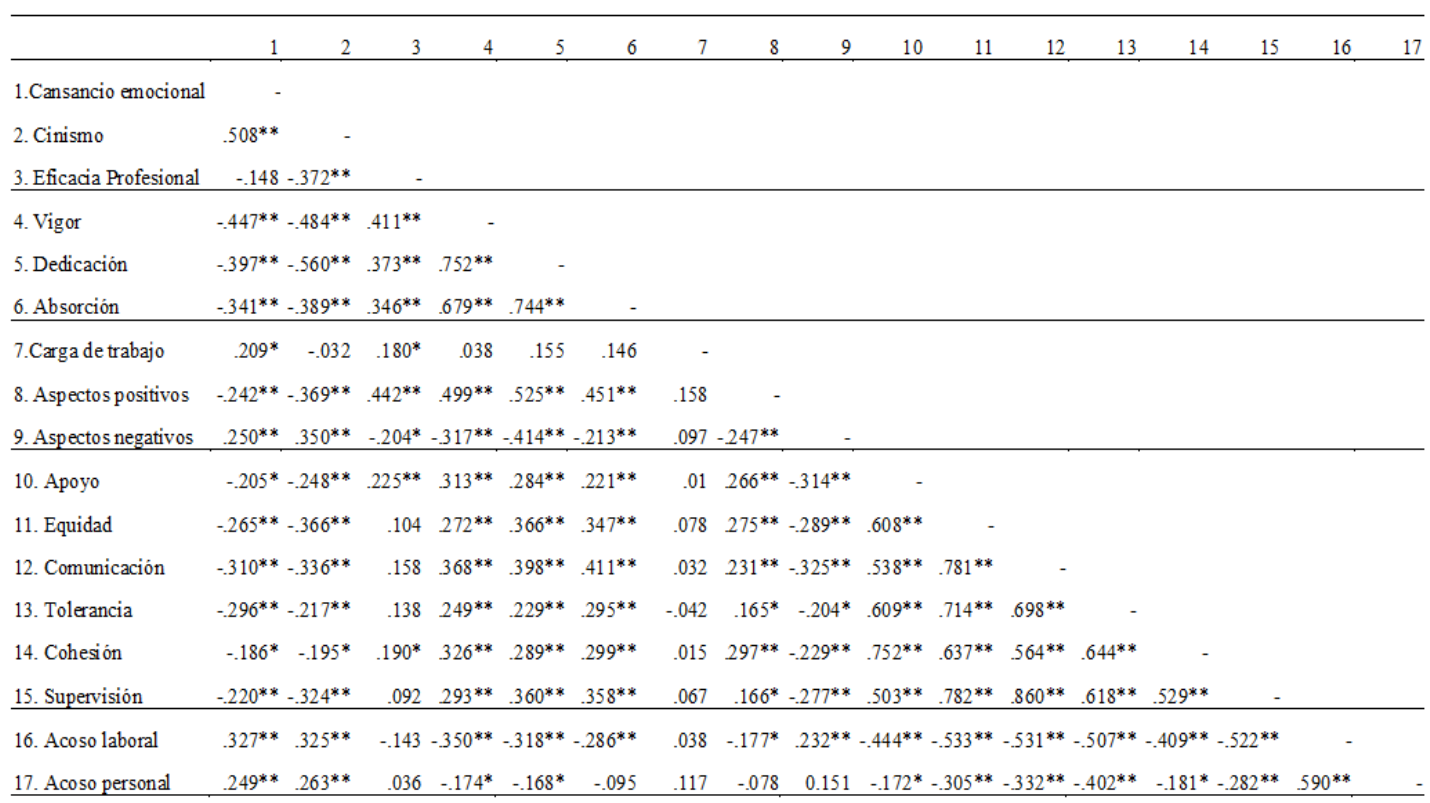

Nota: $* *=\mathrm{p} \leq .01 ; *=\mathrm{p} \leq .05$

En la tabla 2 observamos que las subescalas de burnout y engagement, se encuentran correlacionadas entre sí, destacándose las asociaciones entre dimensiones teóricamente opuestas tales como el cansancio emocional y el vigor $(r=-.447 ; p \leq .01)$, o el cinismo y la dedicación ( $\mathrm{r}=-.560 ; \mathrm{p} \leq .01)$; en ambos casos, con correlaciones inversas o negativas. Por otra parte, la eficacia profesional, como era de esperar, correlacionó positivamente con los factores constitutivos del engagement: vigor $(r=.411 ; \mathrm{p} \leq .01)$, dedicación $(\mathrm{r}=.373 ; \mathrm{p} \leq .01)$ y absorción $(\mathrm{r}=.346 ; \mathrm{p} \leq .01)$. 
Comenzaremos con las principales correlaciones para las subescalas del burnout. En general, el cansancio emocional presentó correlaciones débiles pero significativas de carácter positivo con la percepción de acoso laboral ( $\mathrm{r}=.327 ; \mathrm{p} \leq .01)$ y de carácter inverso, con la variable comunicación $(\mathrm{r}=-.310 ; \mathrm{p} \leq .01)$. El cinismo correlacionó positivamente con el acoso laboral $(\mathrm{r}=.325 ; \mathrm{p} \leq .01)$, con los aspectos negativos de la tarea $(\mathrm{r}=.350$; $\mathrm{p} \leq .01)$ e inversamente con los aspectos positivos $(\mathrm{r}=-.369 ; \mathrm{p} \leq .01)$. En cuanto al clima psicosocial, se observaron correlaciones negativas con las variables equidad $(r=-.366$; $\mathrm{p} \leq .01)$, comunicación $(\mathrm{r}=-.336 ; \mathrm{p} \leq .01)$ y supervisión $(\mathrm{r}=-.324 ; \mathrm{p} \leq .01)$. Por su parte, la eficacia profesional únicamente mostró correlación con los aspectos positivos de la tarea $(\mathrm{r}=.442 ; \mathrm{p} \leq .01)$.

Analicemos ahora las correlaciones para las dimensiones del engagement. El vigor correlacionó directamente con los aspectos positivos de la tarea $(\mathrm{r}=.499 ; \mathrm{p} \leq .01)$, mientras que se vinculó inversamente con los aspectos negativos de la tarea $(\mathrm{r}=-.317 ; \mathrm{p} \leq .01)$ y el acoso laboral $(\mathrm{r}=-.350 ; \mathrm{p} \leq .01)$. Por otra parte, el vigor mostró una débil correlación con las variables apoyo $(\mathrm{r}=.313 ; \mathrm{p} \leq .01)$, comunicación $(\mathrm{r}=.368 ; \mathrm{p} \leq .01)$ y cohesión $(\mathrm{r}=.326$; $\mathrm{p} \leq .01)$. En cuanto a la dimensión dedicación, se observaron correlaciones con los aspectos positivos de la tarea $(\mathrm{r}=.525 ; \mathrm{p} \leq .01)$ y las variables supervisión $(\mathrm{r}=.360 ; \mathrm{p} \leq .01)$, equidad $(\mathrm{r}=.366 ; \mathrm{p} \leq .01)$ y comunicación $(\mathrm{r}=.398 ; \mathrm{p} \leq .01)$ del clima psicosocial. Por otra parte, se observaron correlaciones inversas con los aspectos negativos de la tarea $(r=-.414 ; \mathrm{p} \leq .01)$ y el acoso laboral $(\mathrm{r}=-.318 ; \mathrm{p} \leq .01)$. Por último, la subescala de absorción mostró su más fuerte correlación con los aspectos positivos de la tarea $(\mathrm{r}=.451 ; \mathrm{p} \leq .01)$, y en menor medida correlacionó con comunicación $(\mathrm{r}=.411 ; \mathrm{p} \leq .01)$, equidad $(\mathrm{r}=.347 ; \mathrm{p} \leq .01) \mathrm{y}$ supervisión $(\mathrm{r}=.358 ; \mathrm{p} \leq .01)$.

\section{Análisis por conglomerados}

Finalmente, el análisis por conglomerados de k medias, permitió identificar la existencia de patrones comunes en los participantes y agruparlos en perfiles psicosociales diferenciados. A continuación, presentamos los resultados obtenidos. 
Tabla 3

Centro de conglomerados finales para todas las variables estudiadas

\begin{tabular}{lll} 
& Factor 1 & Factor 2 \\
\hline 1. Cansancio emocional & 0 & 8 \\
2. Cinismo & 0 & 9 \\
3. Eficacia Profesional & 9 & 3 \\
\hline 4. Vigor & 8,57 & 1,43 \\
5. Dedicación & 8,37 & 2,57 \\
6. Absorción & 8,57 & 2,38 \\
\hline 7. Carga de trabajo & 7,6 & 10 \\
8. Aspectos positivos de la tarea & 9 & 2 \\
9. Aspectos negativos de la tarea & 4,29 & 7,71 \\
\hline 10. Apoyo & 2 & 5,33 \\
11. Equidad & 2 & 3,78 \\
12. Comunicación & 10 & 3,5 \\
13. Cohesión & 10 & 3 \\
14. Tolerancia & 10 & 2,5 \\
15. Supervisión & 10 & 4 \\
\hline 16. Acoso laboral & 10 & 3 \\
17. Acoso personal & 10 & 3,5 \\
\hline Porcentajes & $74.2 \%$ & $25.8 \%$ \\
\hline
\end{tabular}

Nota: El primer factor representa el $74.2 \%$ de los casos $(\mathrm{n}=109)$ y el segundo factor representa el $25.8 \%$ de los casos $(n=38)$.

El primer perfil, está compuesto por la mayoría de los participantes $(n=109)$ que representan el $74.2 \%$ de la muestra. Este grupo presenta características psicosociales asociadas principalmente con el engagement y los aspectos positivos del trabajo, por lo que lo denominamos "proactivo". El segundo perfil, compuesto por un $25.8 \%$ de los participantes $(n=38)$, está asociado con los factores negativos de desgaste y baja motivación, por lo cual lo identificamos como grupo "en riesgo". Ambos perfiles psicosociales vinculados al trabajo pueden visualizarse en la siguiente Figura 


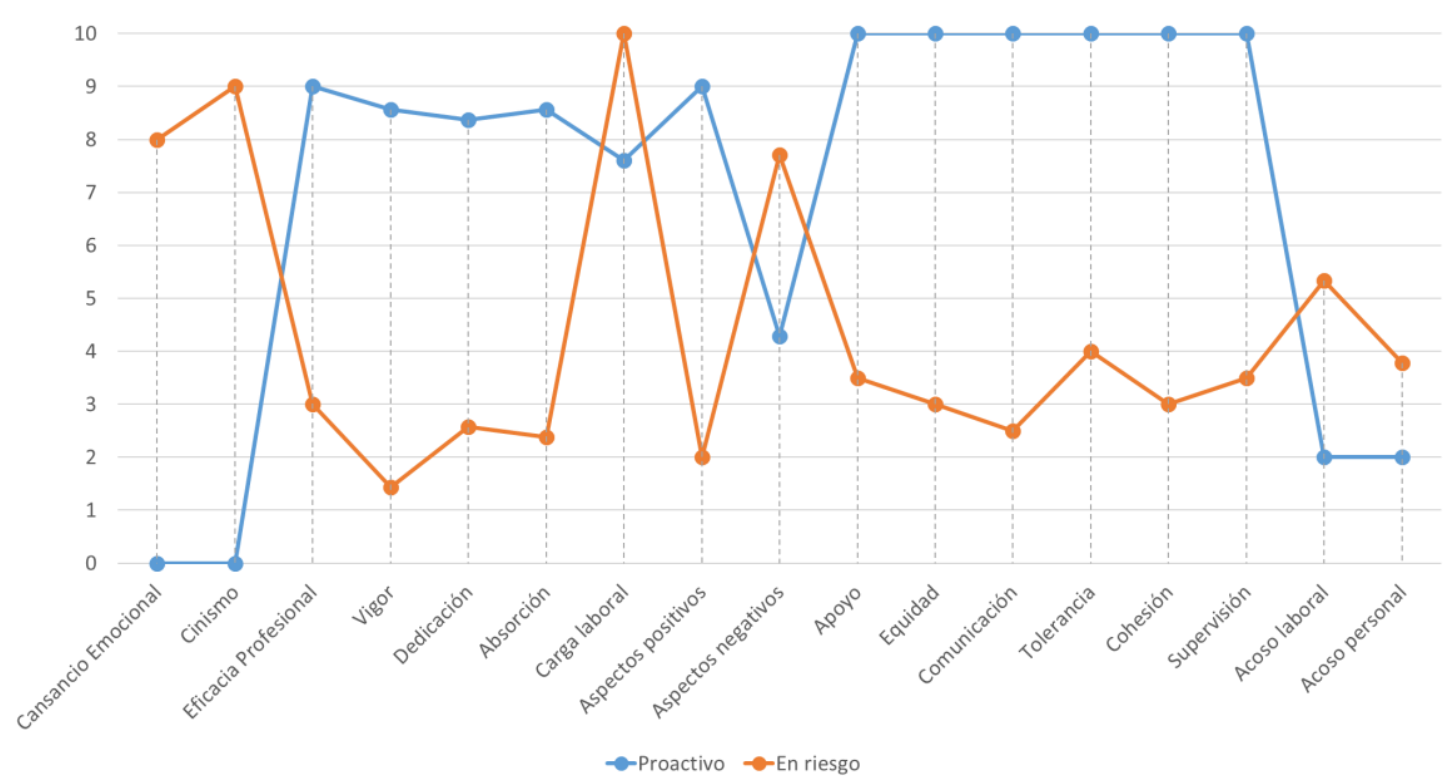

Figura 1. Perfiles psicosociales según conglomerados de k media

Como podemos observar, los empleados del perfil "proactivo" presentan algunas características comunes: a) no poseen indicadores de desgaste (cansancio emocional o cinismo); b) sus niveles de compromiso laboral son muy elevados tanto en vigor, dedicación y absorción en la tarea; c) perciben su carga laboral como alta y acentúan los aspectos positivos de su tarea cotidiana (motivación, autonomía, etc.); d) valoran positivamente los factores que influyen en el clima laboral (apoyo, cohesión, supervisión, equidad, comunicación y tolerancia); y e) perciben baja sensación de acoso laboral o personal.

Por su parte, quienes se incluyen en el grupo "en riesgo" se caracterizan por: a) elevados niveles de cansancio emocional y cinismo (corazón del burnout); b) bajos niveles de compromiso laboral (vigor, dedicación y absorción); c) también perciben su carga laboral como alta, pero destacan los aspectos negativos de su tarea habitual (por ej. aburrimiento, rutina, burocratización); d) subvaloran el clima laboral (apoyo, cohesión, supervisión, equidad, comunicación y tolerancia) y e) presentan mayor sensación de acoso laboral y personal que el otro perfil.

\section{Discusión}

La finalidad de este estudio fue indagar sobre los perfiles psicosociales coexistentes en el ámbito público, a fin de dar cuenta de la complejidad de los contextos 
laborales y las múltiples dinámicas psicosociales que se desarrollan en ellos. Los resultados obtenidos nos permiten arribar a las siguientes conclusiones:

Desde el punto de vista teórico, la aplicación del binomio burnout-engagement demuestra ser un sólido instrumento de análisis que permite indagar sobre los matices que acercan a los trabajadores a experiencias de desgaste o de vinculación positiva con el trabajo (Salanova et al., 2005). Los resultados hallados indican que no es posible hablar en términos de salud o enfermedad en los contextos laborales, sino que en cada organización coexisten distintas experiencias que vinculan de una manera particular a cada trabajador con su entorno y que condicionan las relaciones interpersonales y perfilan la identidad organizativa. En este sentido, nuestros resultados coinciden con la perspectiva dialéctica propuesta por Schaufeli y De Witte (2017), quienes postulan la integración de las experiencias de burnout y engagement, en una misma realidad dual.

En cuanto a sus implicaciones prácticas, lo primero a señalar es el aporte al conocimiento del empleo público, especialmente del staff administrativo, un sector poco investigado en cuanto a riesgos psicosociales y salud ocupacional. A nivel general, una primera lectura descriptiva de los resultados nos daría datos erróneos sobre la población de estudio, ya que atenuaría la intensidad con que se vivencian cada una de las experiencias analizadas. Sin embargo, la identificación de distintos perfiles motivacionales muestra las distintas experiencias positivas o negativas que coexisten en el ámbito laboral a fin de potenciar la construcción de estrategias diferenciadas orientadas a la atención de cada subgrupo: a) para el grupo en riesgo, acentuando propuestas preventivas, capacitaciones o instancias de intervención, buscando subsanar los riesgos psicosociales presentes en el ambiente de trabajo y/o mitigar sus efectos a nivel personal y organizacional; y b) para el grupo proactivo, focalizando en acciones formativas y motivadoras, orientadas a potenciar los recursos personales y organizacionales, que permitan alcanzar altos niveles de motivación vinculación positiva con el trabajo. En otros términos, los resultados fueron insumos para la toma de decisiones que permitieron volver al ámbito laboral con propuestas de mejora. Se espera que dichas propuestas formen parte a futuro de políticas de gestión de recursos humanos sustentables en el tiempo, que permitan elevar los niveles de calidad de vida laboral a partir de la resignificación del trabajo estatal y la revalorización social del empleo público.

En relación con las limitaciones del estudio, cabe señalar que los resultados obtenidos adquieren sentido y significado en su propio contexto de investigación, ya que no es posible generalizarlos a otros escenarios similares. En este sentido, también la 
selección de factores moduladores tiene la misma connotación, ya que se trata de variables que resultaron de peso en análisis previos, pero que podrían variar de un contexto a otro.

Este puntapié inicial, nos invita a seguir profundizando en el análisis integral de los contextos laborales desde su complejidad constitutiva, identificando las distintas experiencias positivas y negativas que condicionan el bienestar de los empleados en los organismos públicos. Si bien la coexistencia de distintos perfiles psicosociales, puede ser fuente de conflicto, incertidumbre y disidencia, esto es lo que hace activas, dinámicas y vitales a las organizaciones, y consecuentemente, con posibilidades de cambio. 


\section{Referencias}

Aron, A. \& Aron, E. (2002). Estadística para Psicología. Prentice Hall/Pearson Educación.

Ayuso Marente, J. A. \& Gillén Gestoso, C. L. (2008). Burnout y mobbing en enseñanza secundaria. Revista Complutense de Educación, 19(1), 157-174. https://dialnet.unirioja.es/servlet/articulo?codigo $=2555685$

Bakker, A. B. \& Demerouti, E. (2013). La teoría de las demandas y los recursos laborales. Revista de Psicología del Trabajo y de las Organizaciones, 29(3), 107-115. https://doi.org/10.5093/tr2013a16

Bakker, A. B. \& Rodríguez-Muñoz, A. (2012). Introducción a la psicología de la salud ocupacional positiva. Psicothema, 24(1), 62-65. https://reunido.uniovi.es/index.php/PST/article/view/9104

Boada i Grau, J., Diego Vallejo, R. D. \& Agulló Tomás, E. (2004). El burnout y las manifestaciones psicosomáticas como consecuentes del clima organizacional y de la motivación laboral. Psicothema, 16 (1), 125-131. https://reunido.uniovi.es/index.php/PST/article/view/8198

Cifre, E.; Salanova, M. \& Ventura, M. (2009). Demandas y recursos del ambiente de trabajo. En Salanova, M. Psicología de la Salud Ocupacional. Síntesis.

Fidalgo, Á. M. \& Piñuel, I. (2004). La escala Cisneros como herramienta de valoración del mobbing. Psicothema, 16(4), 615-624. https://digibuo.uniovi.es/dspace/handle/10651/26722

García-Izquierdo, M., Llor Esteban, B., León García-Izquierdo, A. \& Ruiz Hernández, J. A. (2006). Bienestar psicológico y mobbing en una muestra de profesionales de los sectores educativo y sanitario. Revista de Psicología del Trabajo y de las Organizaciones, 22(3), 381-395. https://journals.copmadrid.org/jwop/art/a64c94baaf368e1840a1324e839230d $\underline{\mathrm{e}}$

Gil Monte, P. (2007). El síndrome de quemarse por el trabajo (burnout) como fenómeno transcultural. Información Psicológica, (91-92), 4-11. https://dialnet.unirioja.es/servlet/articulo? codigo $=2551783$

Gil Monte, P. (2012). Riesgos psicosociales en el trabajo y salud ocupacional. Revista Peruana de Medicina Experimental y Salud Pública, 29(2), 237-241. https://doi.org/10.17843/rpmesp.2012.292.348

Gil-Monte, P., López-Vílchez, J., Llorca-Rubio, J. L. \& Sánchez Piernas, J. (2016). Prevalencia de riesgos psicosociales en personal de la administración de justicia de la Comunidad Valenciana (España). Liberabit, 22(1), 7-19. https://doi.org/10.24265/liberabit.2016.v22n1.01

Golembiewski, R., Munzenrider, R. \& Carter, D. (1983). Progresive phases of burnout and their worksite covariants. Journal of Applied Behavioral Science, 19 (4), 464-481.

González Trijueque, D. \& Delgado Marina, S. (2008). Acoso psicológico en el lugar de trabajo, burnout y psicopatología. Un estudio piloto con el BSI y el MBI. Boletín de Psicología, (94), 47-56. https://dialnet.unirioja.es/servlet/articulo?codigo $=2887722$

Leiter, M. \& Maslach, C. (1999). Six areas of worklife: a model of the organizational context of burnout. Journal of Health and Human Services Administration, 472-489. 
Leiter, M. \& Maslach, C. (2016). Latent burnout profiles: A new approach to understanding the burnout experience. Burnout Research, 3(4), 89-100. http://dx.doi.org/10.1016/j.burn.2016.09.001

Leiter, M. \& Maslach, C. (2017). Burnout and engagement: contributions to a new vision. Burnout research, 5, 55-57. http://dx.doi.org/10.1016/j.burn.2017.04.003

Leon, M. R., Halbesleben, J. R. \& Paustian-Underdahl, S. C. (2015). A dialectical perspective on burnout and engagement. Burnout Research, 2(2-3), 87-96. https://doi.org/10.1016/j.burn.2015.06.002

Marsollier, R. (2018). Empleo Público y Desgaste Profesional. Un Análisis desde las Características de la Tarea. Revista Interamericana de Psicología Ocupacional, 35(2), 80-89. https://doi.org/10.21772/ripo.v35n2a03

Martínez Gamarra, M. \& Ros Mar, R. (2010). De la calidad de la vida laboral a los riesgos Psicosociales: evaluación de la calidad de vida laboral. Acciones e investigaciones sociales, (28), 5-55. https://doi.org/10.26754/ojs_ais/ais.201028480

Maslach, C. \& Leiter, M. (1997). The truth about burnout: How organizations cause personal stress and what to do about it. San Francisco, CA: Jossey-Bass.

Maslach, C. \& Leiter, M. (2008). Early Predictors of Job Burnout and Engagement. Journal of Applied Psychology, 93(3), 498-512. https://doi.org/10.1037/0021-9010.93.3.498

Maslach, C. (2009). Comprendiendo el burnout. Ciencia y Trabajo, 11(32), 37-43. https://dialnet.unirioja.es/servlet/articulo?codigo $=3013987$

Maslach, C., Jackson, S. \& Leiter, M. (1996). Maslach Burnout Inventory Manual. Consulting Psychologists Press.

Meseguer, M., Soler M.I., García-Izquierdo, M., Sáez, M.C. \& Sánchez, J. (2007). Los factores psicosociales de riesgo en el trabajo como predictores del mobbing. Psicothema, 19(2), 225-230. https://reunido.uniovi.es/index.php/PST/article/view/8570

Moreno Jiménez, B., Rodríguez Muñoz, A., Garrosa Hernández, E. \& Morante Benadero, M. E. (2005). Antecedentes organizacionales del acoso psicológico en el trabajo: un estudio exploratorio. Psicothema, 17(4), 627-632. https://reunido.uniovi.es/index.php/PST/article/view/8322

Olmedo, M. \& González, P. (2006). La violencia en el ámbito laboral: la problemática conceptuación del mobbing, su evaluación, prevención y tratamiento. Acción psicológica, 4(2), 107-128. https://dialnet.unirioja.es/servlet/articulo?codigo $=2904108$

Orgambídez-Ramos, A., Pérez-Moreno, P. J. \& Borrego-Alés, Y. (2015). Estrés de rol y satisfacción laboral: examinando el papel mediador del engagement en el trabajo. Revista de Psicología del Trabajo y de las Organizaciones, 31(2), 69-77. https://doi.org/10.1016/j.rpto.2015.04.001

Pecino, V., Mañas, M. A., Díaz-Fúnez, P. A., Aguilar-Parra, J. M., Padilla-Góngora, D. \& López-Liria, R. (2019). Organisational Climate, Role Stress, and Public Employees' Job Satisfaction. International Journal of Environmental Research and Public Health, 16(10), 1792. https://doi.org/10.3390/ijerph16101792

Pérez, J. P. (2013). Efecto del burnout y la sobrecarga en la calidad de vida en el trabajo. Estudios gerenciales, 29(129), 445-455. https://doi.org/10.1016/j.estger.2013.11.010 
Rodríguez Muñoz, M. F., Osona Miguel, J. A., Domínguez Martín, A. L. \& Comeche Moreno, M. I. (2009). Mobbing: una propuesta exploratoria de intervención. International Journal of Psychology and Psychological Therapy, 9(2), 177-188. https://dialnet.unirioja.es/servlet/articulo?codigo=2992444

Rothlin, P. \& Werder, P. (2011). El nuevo síndrome laboral Boreout: Recupera la motivación. Debolsillo.

Sáez, M., García-Izquierdo, M. \& LLor, B. (2003). Validación de la escala NAQ de Einarsen y Raknes (1997) sobre acoso psicológico en el trabajo (mobbing). VIII National Congress of Social Psychology, in the Symposium of Mobbing: una perspectiva psicosocial. Torremolinos, Málaga.

Salanova, M. \& Llorens, S. (2008). Estado actual y retos futuros en el estudio del burnout. Papeles del Psicólogo, 29(1), 59-67. http://www.redalyc.org/html/778/77829108/

Salanova, M., Bresó, E. \& Schaufeli, W. (2005). Hacia un modelo espiral de las creencias de eficacia en el estudio del burnout y del engagement. Ansiedad y estrés, 11(2-3), 215-231. https://www.wilmarschaufeli.nl/publications/Schaufeli/243.pdf

Salanova, M., Martínez, I. M. \& Llorens, S. (2014). Una mirada más “positiva” a la salud ocupacional desde la psicología organizacional positiva en tiempos de crisis: aportaciones desde el equipo de investigación WoNT. Papeles del psicólogo, 35(1), 22-30. https://dialnet.unirioja.es/servlet/articulo?codigo $=4600665$

Salanova, M., Schaufeli, W., Llorens, S., Peiró, J. \& Grau, R. (2000). Desde el" burnout" al" Engagement". Una nueva perspectiva. Revista de Psicología del Trabajo y las Organizaciones, 16(2), 117-134. https://journals.copmadrid.org/jwop/art/7c590f01490190db0ed02a5070e20f0 $\underline{1}$

Schaufeli, W. \& Salanova, M. (2014). Burnout, boredom and engagement at the workplace. En M. Peeters, J. de Jonge \& T. Taris (Eds) People at work: An introduction to contemporary work psychology, 293-320.

Schaufeli, W. \& De Witte, H. (2017). Outlook Work Engagement: Real and Redundant! Burnout Research, 5, 58-60. https://doi.org/10.1016/j.burn.2017.06.002

Schaufeli, W., Leiter, M. P. \& Maslach, C. (2009). Burnout: 35 years of research and practice. Career Development International, 14(3), 204-220. https://doi.org/10.1108/13620430910966406

Schaufeli, W., Leiter, M., Maslach, C. \& Jackson, S. (1996). The Maslach Burnout Inventory: General Survey (MBI-GS). En: Maslach, C., Jackson, C. \& Leiter, M. (Eds.), The Maslach Burnout Inventory Manual ( $3^{\mathrm{a}}$ ed., pp. 19-26). Consulting Psychologists Press.

Schaufeli, W., Salanova, M., González-Romá, V. \& Bakker, A. (2002). The measurement of burnout and engagement: A confirmatory factor analytic approach. Journal of Happiness Studies, 3, 71-92. https://link.springer.com/article/10.1023/A:1015630930326

Seligman, M. \& Csikszentmihalyi, M. (2000). Positive Psychology: An Introduction. American Psychologist, 55(1), 5-14. https://doi.org/10.1037/0003066X.55.1.5 
Sonnentag, S. (2017). A task-level perspective on work engagement: A new approach that helps to differentiate the concepts of engagement and burnout. Burnout research, 5, 12-20.

Sonnentag, S. \& Kühnel, J. (2016). Coming back to work in the morning: Psychological detachment and reattachment as predictors of work engagement. Journal of Occupational Health Psychology, 21(4), 379-390. https://doi.org/10.1037/ocp0000020

Torrente, P., Acosta, H., Llorens, S. \& Salanova, M. (2017). Prácticas organizacionales saludables. Revista Peruana de Psicología y Trabajo Social, 2(1), 107-120. http://revistas.uigv.edu.pe/index.php/psicologia/article/view/79/87

Winnubst, J. (1993). Organizational structure, social support, and burnout. En W.B. Schaufeli, C. Maslach y T. Marek (Eds.), Profesional burnout: Recent developments in theory and research. Londres: Taylor y Francis, 151-162. 